\title{
Dysregulated Expression of MITF in Subsets of Hepatocellular Carcinoma and Cholangiocarcinoma
}

\author{
Nattakarn Nooron, ${ }^{1,2}$ Koji Ohba, ${ }^{1, *}$ Kazuhisa Takeda, ${ }^{1, \dagger}$ Shigeki Shibahara ${ }^{1,3}$ and \\ Anchalee Chiabchalard ${ }^{2}$

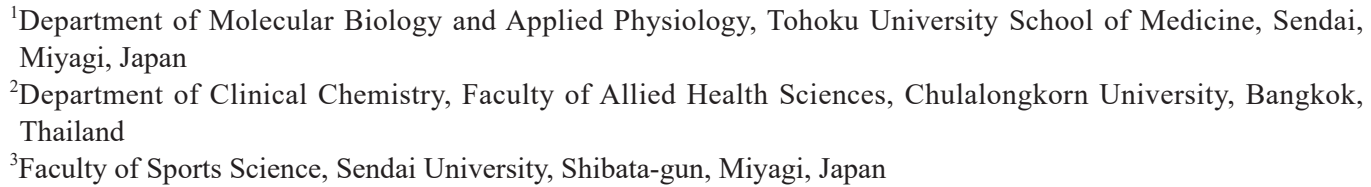

Cholangiocarcinoma represents the second most common primary liver tumor after hepatocellular carcinoma. Mahanine, a carbazole alkaloid derived from Murraya koenigii (Linn.) Spreng, has been used as folk medicine in Thailand, where the liver fluke-associated cholangiocarcinoma is common. The expression of microphthalmia-associated transcription factor (MITF) is maintained at immunohistochemically undetectable levels in hepatocytes and cholangiocytes. To explore the regulation of MITF expression in the liver, we immunohistochemically analyzed the MITF expression using hepatocellular carcinoma and cholangiocarcinoma specimens of the human liver cancer tissue array. MITF immunoreactivity was detected in subsets of hepatocellular carcinoma (6 out of 38 specimens; $16 \%$ ) and cholangiocarcinoma (2/7 specimens; 29\%). Moreover, immunoreactivity for glioma-associated oncogene 1 (GLI1), a transcription factor of the Hedgehog signaling pathway, was detected in $55 \%$ of hepatocellular carcinoma $(21 / 38$ specimens) and $86 \%$ of cholangiocarcinoma (6/7 specimens). Importantly, MITF was detectable only in the GLI1-positive hepatocellular carcinoma and cholangiocarcinoma, and MITF immunoreactivity is associated with poor prognosis in patients with hepatocellular carcinoma. Subsequently, the effect of mahanine was analyzed in HepG2 human hepatocellular carcinoma and HuCCT1 and KKU-100 human cholangiocarcinoma cells. Mahanine $(25 \mu \mathrm{M})$ showed the potent cytotoxicity in these hepatic cancer cell lines, which was associated with increased expression levels of MITF, as judged by Western blot analysis. MITF is overexpressed in subsets of hepatocellular carcinoma and cholangiocarcinoma, and detectable MITF immunoreactivity is associated with poor prognosis in patients with hepatocellular carcinoma. MITF expression levels may be determined in hepatic cancer cells by the balance between the Hedgehog signaling and the cellular stress.

Keywords: cholangiocarcinoma; GLI1; Hedgehog signaling; hepatocellular carcinoma; mahanine Tohoku J. Exp. Med., 2017 August, 242 (4), 291-302. C 2017 Tohoku University Medical Press

\section{Introduction}

Microphthalmia-associated transcription factor (Mitf) contains a basic helix-loop-helix and a leucine-zipper (bHLH-LZ) structure, and it has been established as a key regulator for development of melanocytes and the retinal pigment epithelium (RPE) (Hodgkinson et al. 1993; Hughes et al. 1993; Steingrimsson et al. 1994; Hozumi et al. 2012). However, subsequent studies have suggested the pleiotropic functions of Mitf in various organs, including liver (Smith et al. 1994), pancreas (Mazur et al. 2013), kidney (Ohba et al. 2016), lung (Takeda et al. 2007), olfactory bulb (Ohba et al. 2015), and frontal cortex (Takeda et al. 2014; Ohba et al. 2016). In particular, the Mitf vitiligo mouse, $\mathrm{Mitf}^{\mathrm{vit}} / \mathrm{Mitf}^{\mathrm{vit}}$, is characterized by retinal degeneration and the accumulation of retinyl palmitate in RPE and the liver (Smith et al. 1994). The Mitf ${ }^{\text {vit }}$ protein carries the Asp222Asn substitution (Steingrimsson et al. 1994) and its mutant protein is predicted to show the decrease in Mitf function (Yasumoto et al. 2002). Thus, Mitf may be involved in the metabolic function of hepatocytes and/or cholangiocytes. However, the immunohistochemical analysis of the mouse liver

Received June 23, 2017; revised and accepted July 20, 2017. Published online August 10, 2017; doi: 10.1620/tjem.242.291.

Correspondence: Anchalee Chiabchalard, Department of Clinical Chemistry, Faculty of Allied Health Sciences, Chulalongkorn

University, Bangkok 10330, Thailand.

e-mail: Anchalee.c@ chula.ac.th

Present addresses:

*Department of Endocrinology and Applied Medical Science, Tohoku University Graduate School of Medicine, Sendai, Miyagi, Japan.

${ }^{\dagger}$ Division of Molecular Physiology and Metabolism, Tohoku University School of Medicine, Sendai, Miyagi, Japan. 
revealed that Mitf immunoreactivity was detected only in Kupffer cells, but not detectable in hepatocytes, cholangiocytes, and other cell types (Ohba et al. 2016). In addition, the mRNA levels of MITF, a human orthologue of Mitf, were marginally detected in the human liver (Samatiwat et al. 2016). We therefore hypothesize that the expression of Mitf/MITF may be maintained at the low level in hepatocytes and cholangiocytes.

Cholangiocarcinoma is a malignant tumor that is originated from the biliary epithelial cell, and is the second most common primary liver tumor, accounting for about $10-15 \%$ after hepatocellular carcinoma (Shaib and El-Serag 2004). Both types of hepatic cancer are characterized by poor prognosis. We reported that cyclopamine, an inhibitor of Hedgehog signaling, increased the expression levels of MITF in human hepatocellular carcinoma and cholangiocarcinoma cell lines (Samatiwat et al. 2016). Importantly, cyclopamine is a natural teratogen, contained in the plant Veratrum californicum, and it causes craniofacial birth defects in sheep, such as cyclopia (Lee et al. 2014). Cyclopamine acts on a signal transducer, Smoothened, located in plasma membrane (Lee et al. 2014) to inhibit the Hedgehog signaling. In the presence of a Hedgehog ligand, Smoothened enhances nuclear translocation of gliomaassociated transcription factors, such as glioma-associated oncogene 1 (GLI1) (Varjosalo and Taipale 2008), thereby mediating the Hedgehog signal. Moreover, GLI1 is expressed in the normal bile duct (Kiesslich et al. 2014) and is overexpressed in hepatocellular carcinoma (Dugum et al. 2016) and cholangiocarcinoma (Kiesslich et al. 2014; Riedlinger et al. 2014), suggesting that the Hedgehog signaling pathway may be activated in hepatic cancer cells. Considering the cyclopamine-mediated induction of MITF in hepatic cancer cell lines (Samatiwat et al. 2016), we have hypothesized that the Hedgehog signaling may influence MITF expression in the liver.

As a first step to study the regulation of MITF in the liver, we analyzed its expression profile in human hepatocellular carcinoma and cholangiocarcinoma. We thus show that MITF immunoreactivity is detected in subsets of human hepatocellular carcinoma and cholangiocarcinoma specimens that also express GLI1. Subsequently, we found the increase in MITF expression in human hepatic cancer cells treated with mahanine, a carbazole alkaloid identified in the leaves of Murraya koenigii (Linn.) Spreng (curry leaf) (Roy et al. 2004; Utaipan et al. 2017). This plant is used as folk medicine in Thailand, where the liver flukeassociated cholangiocarcinoma is common (Shaib and El-Serag 2004). In fact, the infection with the liver fluke (Opisthorchis viverrini) is a well-known risk factor of cholangiocarcinoma in Thailand (Haswell-Elkins et al. 1992; Shin et al. 2010).

In summary, MITF is over-expressed in subsets of hepatocellular carcinoma and cholangiocarcinoma. Conversely, MITF expression may be maintained at the immunologically undetectable level in most of hepatic can- cer, similar to the normal liver. A better understanding the regulation of MITF expression may contribute to developing a novel anti-hepatic cancer agent.

\section{Materials and Methods}

Materials

Human liver cancer tissue array (LV484) was purchased from US Biomax, Inc. (Rockville, MD, USA). GANT61, Gli-ANTagonist (Lauth et al. 2007), was purchased from Wako (Osaka, Japan) and was dissolved in dimethyl sulfoxide (DMSO) (Merck, Darmstadt, Germany). MTT [3-(4,5-dimethylthiazol-2-yl)-2,5-diphenyltetrazolium bromide] was from Nacalai tesque, Japan, and Difco ${ }^{\mathrm{TM}}$ trypsin 250 was from BD Becton (Sparks, MD, USA). EDTA was from Dojindo Molecular Technologies. Trypan blue staining was from Sigma-Aldrich (St. Louis, MO, USA). Mahanine [3,11-dihydro3,5-dimethyl-3-(4-methyl-3-pentenyl)], a carbazole alkaloid, was kindly provided by Prof. Apichart Suksamran, Department of Chemistry, Faculty of Sciences, Ramkhamheng University, Bangkok, Thailand. Mahanine was dissolved in dimethyl sulfoxide (DMSO) at $100 \mathrm{mM}$ as stock solution and further diluted with medium for cell treatment.

\section{Immunohistochemical analysis of human hepatic cancer arrays}

The liver cancer tissue array contained 40 cases of hepatocellular carcinoma and 8 cases of cholangiocarcinoma (LV484, US Biomax, Inc.), and two arrays of the same lot were used for analyzing the expression of MITF and GLI1. However, one hepatocellular carcinoma specimen and one cholangiocarcinoma specimen were excluded, because one of the tissue specimens derived from a same patient was peeled from a slide glass during the immunohistochemical procedure with either anti-MITF antibody or anti-GLI1 antibody. In addition, one hepatocellular carcinoma specimen was excluded, because its grade in Pathology Diagnosis was not available. Accordingly, the remaining 38 hepatocellular carcinoma cases and 7 cholangiocarcinoma cases were analyzed. Anti-MITF polyclonal antibody was produced in rabbits using His-Tag-MITF-M as an antigen (Fuse et al. 1999). MITF-M represents the melanocyte-specific isoform of MITF (Yasumoto et al. 1994). The antibody thus obtained recognizes a common region that is shared by multiple MITF isoforms, including MITF-M and widely expressed MITF-A and MITF-H (Fuse et al. 1999). Importantly, this antibody has been successfully used in immunohistochemical analysis (Ohba et al. 2015, 2016). Dilutions of primary antibodies were 1:400 for anti-MITF and 1:100 for anti-GLI1 (BIOSS, Woburn, MA, USA). For simplicity, the presence of detectable immunoreactivity was defined as positive, irrespective of the staining intensity, while the absence of detectable immunoreactivity was defined as negative. In case of positive specimens, almost all cancer cells were reactive with a given polyclonal antibody (anti-MITF or anti-GLI1 antibody). As negative control, the tissue sections were incubated with normal rabbit $\operatorname{IgG}$ (DAKO), instead of a primary antibody (data not shown).

\section{Cell culture}

Human cholangiocarcinoma cell lines, KKU-100 and HuCCT1, were obtained from JCRB Cell Bank (Osaka, Japan) and RIKEN Cell Bank (Tsukuba, Japan), respectively. KKU-100 cells were cultivated in Dulbecco's Modified Eagle's Medium (DMEM) containing 10\% fetal bovine serum (FBS), and HuCCT1 cells were cultivated in RPMI1640 medium containing 10\% FBS. HepG2 hepatocellular car- 
cinoma cells were cultivated in DMEM containing 10\% FBS.

Assessment of cytotoxicity

Cytotoxicity of GANT61 or mahanine was determined in KKU100 and HuCCT1 cholangiocarcinoma cells and HepG2 hepatocellular carcinoma cells by MTT assay, which is the measure of enzyme activity in mitochondria that reduces 3-(4, 5-dimethylthiazol-2-yl)2,5-diphenyltetrazolium bromide (MTT) to purple formazan. In brief, cells were seeded and grown in 96-well flat bottom culture plates at a density of $1 \times 10^{4}$ cells/well for $24 \mathrm{~h}$ before treatment with varying concentrations of GANT61 for $48 \mathrm{~h}$ or mahanine for $24 \mathrm{~h}$. After treatment, culture medium was removed and fresh medium was added. MTT at a final concentration of $1 \mathrm{mg} / \mathrm{ml}$ was added into each well in the 96 -well plate and incubated for $4 \mathrm{~h}$ in a humidified atmosphere at $37^{\circ} \mathrm{C}$ under $5 \% \mathrm{CO}_{2}$. After the medium was removed, acidic isopropanol $(100 \mu \mathrm{l})$ was added to dissolve the formazan crystal. The amount of formazan was determined based on absorbance at $570 \mathrm{~nm}$, using a plate reader (FlexStation 3 Microplate Reader, Molecular Devices). After subtraction of background, cell viability was expressed as percentage relative to control that was designated as $100 \%$.

In another series of experiments, cells were seeded in $60-\mathrm{mm}$ culture dishes and allowed to grow overnight (80-90\% confluence), then incubated with vehicle (DMSO), GANT61 $(10$ or $20 \mu \mathrm{M})$ or mahanine (up to $25 \mu \mathrm{M}$ ) in fresh medium containing $10 \%$ FBS for 24 or $48 \mathrm{~h}$. Treated cells were washed twice with ice-cold PBS, detached with cell scrapers, and pelleted by centrifugation at $5,000 \mathrm{~g}$ at $4^{\circ} \mathrm{C}$ for $3 \mathrm{~min}$. Whole cell lysates were prepared, as described previously (Samatiwat et al. 2016), and cell extracts were collected after centrifuged at $14,000 \mathrm{~g}$ for $15 \mathrm{~min}$ at $4^{\circ} \mathrm{C}$. Cell extracts $(30 \mu \mathrm{g}$ protein per lane) were subjected to Western blot analysis, as detailed below. The relative intensity of a specific protein band was assessed with the intensity of $\beta$-actin (loading control).

\section{Western blot analysis}

Whole cell lysates were prepared from KKU-100 and HuCCT1 cholangiocarcinoma cells and HepG2 hepatocellular carcinoma cells. Cell extracts (30 $\mu \mathrm{g}$ protein per lane) were fractionated by SDSPAGE and blotted to a polyvinylidene fluoride membrane (Immobilon $^{\mathrm{TM}}-\mathrm{P}$, Millipore Corporation) in the buffer containing 20\% methanol, $48 \mathrm{mM}$ Tris, $39 \mathrm{mM}$ glycine, and $0.037 \%$ SDS. The membranes were treated with Tris-buffered saline (TBS) with $0.1 \%$ Tween 20 (TBS-T), containing 5\% non-fat dried milk for $1 \mathrm{~h}$ at room temperature, exposed with each antibody in TBS-T for $2 \mathrm{~h}$ at room temperature, and were washed three times each for $10 \mathrm{~min}$ in TBS-T at room temperature. The antibodies used were mouse monoclonal antiMITF antibody (C5) (NeoMarkers, Fremont, CA), anti-heme oxygenase (HO)-1 antibody (ADI-SPA-896, Enzo Life science, Farmindale, NY, USA), and anti- $\beta$-actin antibody (Sigma-Aldrich, St. Louis, MO). Finally, immunoreactive proteins were detected with a Western blot kit (Millipore Corporation). Horseradish peroxidase (HRP)conjugated donkey anti-rabbit immunoglobulin (NA9340) and HRPconjugated sheep anti-mouse immunoglobulin (NA931) were from GE Healthcare (Buckinghamshire, UK).

\section{Statistical analysis}

The cytotoxicity experiments were performed at least three independent times, and all data were expressed as the mean \pm standard deviation (SD). Statistical significance of the results was ana- lyzed using Student t-test. A p value $<0.05$ was considered significant.

\section{Results \\ Expression profiles of MITF in hepatocellular carcinoma}

To explore the hitherto unknown role of MITF in the liver, we immunohistochemically analyzed the expression profiles of MITF in hepatocellular carcinoma of the human liver cancer tissue array. Because we used two separate tissue arrays containing the same series of cancer specimens, it is not necessarily possible to analyze the consecutive tissue sections. GLI1 expression was also analyzed as a poor prognosis marker of hepatocellular carcinoma (Che et al. 2012). Fig. 1 shows representative tissue sections of one hepatocellular carcinoma that was negative for both MITF and GLI1 immunoreactivities (tissue sections of panel A) and one sample that was positive for both MITF and GLI1 immunoreactivities (tissue sections of panel B). Notably, MITF and GLI1 immunoreactivities were consistently detected in cytoplasm, but not detectable in cell nuclei.

The immunohistochemical analysis of 38 hepatocellular carcinoma specimens revealed the two groups of carcinoma (Table 1): cancer cells with undetectable MITF immunoreactivity $(\mathrm{n}=32,84 \%)$ and those with detectable MITF immunoreactivity $(\mathrm{n}=6,16 \%)$. Thus, MITF was expressed below the immunologically detectable levels in most cases of hepatocellular carcinoma. Importantly, three of the six MITF-positive hepatocellular carcinomas were categorized as poorly differentiated histology (50\%), whereas eight of 32 MITF-negative hepatocellular carcinomas $(25 \%)$ were categorized as poorly differentiated histology (Table 1). Thus, detectable MITF immunoreactivity is associated with poor prognosis in patients with hepatocellular carcinoma. Incidentally, the hepatocellular carcinoma specimens of female patients were negative for MITF immunoreactivity $(\mathrm{n}=4$; Table 1$)$, although there was no sex difference in the positive rate for MITF immunoreactivity.

On the other hand, GLI1 immunoreactivity was detected in 55\% of hepatocellular carcinoma specimens (21 out of 38 specimens) and undetectable in $45 \%$ specimens (17 out of 38 specimens; Table 2). Moreover, GLI1positive hepatocellular carcinoma specimens showed poorly differentiated phenotypes (Table 2), whereas all of GLI1negative hepatocellular carcinoma specimens showed moderately differentiated phenotypes. These results are consistent in part with the implication of GLI1 expression as a poor prognosis marker (Che et al. 2012). The positive rate for GLI1 immunoreactivity was significantly lower in female patients than the rate in male patients $(P=0.03$, Table 2). In fact, all of the four specimens of female patients were negative for both MITF and GLI1 immunoreactivities (Tables 1 and 2).

Importantly, MITF immunoreactivity was detected only in GLI1-positive hepatocellular carcinoma specimens (6 out of 21 GLI1-positive specimens, 29\%; Table 3), sug- 


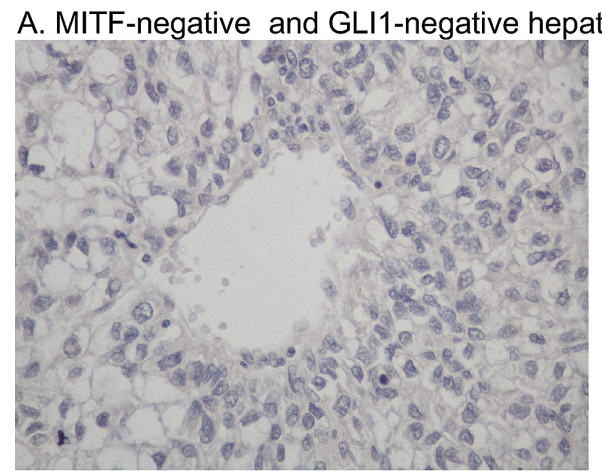

MITF

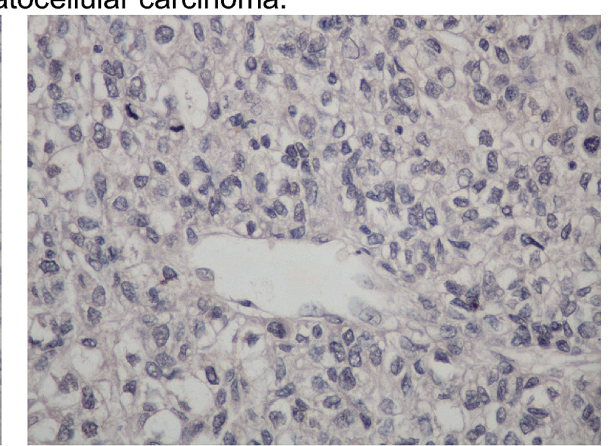

GLI1

B. MITF-positive and GLI1-positive hepatocellular carcinoma.

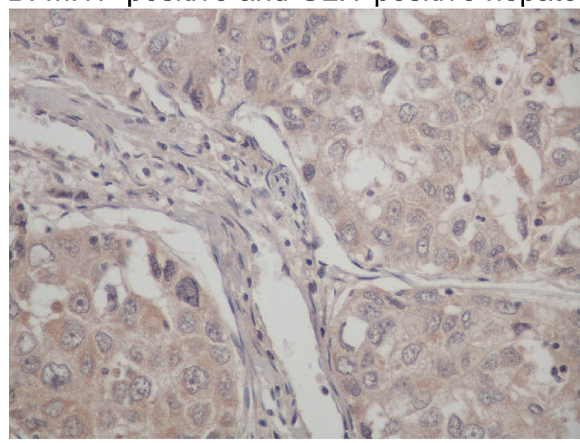

MITF

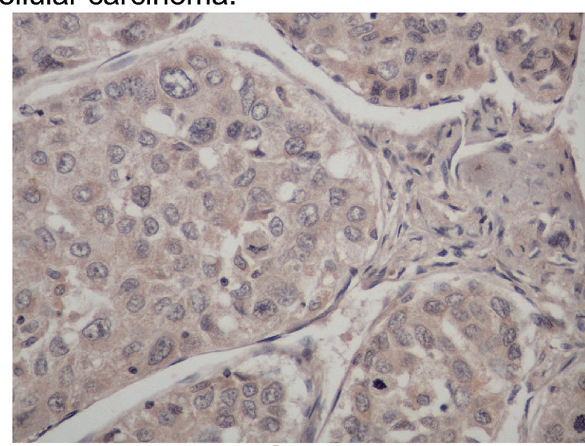

GLI1

Fig. 1. Expression profiles of immunoreactive MITF and GLI1 in hepatocellular carcinoma.

(A) One representative hepatocellular carcinoma specimen that is negative for MITF immunoreactivity (left) and GLI1 immunoreactivity (right) (magnification: $\times 400$ ), out of 17 double-negative specimens. The presented hepatocellular carcinoma specimen was categorized as grade 2 (moderately differentiated) (Tables 1 and 2); namely, cells appear slightly different than normal. (B) One representative hepatocellular carcinoma specimen that is positive for MITF immunoreactivity (left) and GLI1 immunoreactivity (right) (magnification: $\times 400$ ), out of six double-positive specimens. The presented hepatocellular carcinoma specimen was categorized as grade 3 (poorly differentiated) (Tables 1 and 2); namely, cells appear abnormal. Note that MITF immunoreactivity was detected only in GLI1-positive hepatocellular carcinoma.

Table 1. MITF expression in Hepatocellular Carcinoma.

\begin{tabular}{|c|c|c|c|}
\hline & \multicolumn{2}{|c|}{ MITF immunoreactivity } & \multirow{2}{*}{$P$ value } \\
\hline & positive $(n=6)$ & negative $(n=32)$ & \\
\hline Age (years)* & $52 \pm 12$ & $52 \pm 11$ & 0.97 \\
\hline \multicolumn{4}{|l|}{ Sex } \\
\hline Male & 6 & 28 & \\
\hline Female & 0 & 4 & 0.99 \\
\hline \multicolumn{4}{|l|}{ Stage } \\
\hline I & 0 & 0 & \\
\hline II & 3 & 16 & \\
\hline III & 3 & 16 & 0.99 \\
\hline \multicolumn{4}{|c|}{ Histological grade } \\
\hline 1 (well) & 2 & 0 & \\
\hline 2 (moderate) & 1 & 25 & \\
\hline 3 (poor) & 3 & 7 & 0.0006 \\
\hline
\end{tabular}

*Data (years) are presented as the mean \pm SD. All other values represent the number of patients. $\mathrm{P}$ values $<0.05$ are considered to be significant, indicated bold. 
Table 2. GLI1 expression in Hepatocellular Carcinoma.

\begin{tabular}{lccc}
\hline & \multicolumn{2}{c}{ GLI1 immunoreactivity } & \multirow{2}{*}{ P value } \\
\cline { 2 - 3 } & positive $(\mathbf{n}=\mathbf{2 1})$ & negative $(\mathbf{n}=\mathbf{1 7})$ & \\
\hline Age (years)* & $52 \pm 13$ & $52 \pm 9$ & 0.60 \\
Sex & & & \\
$\quad$ Male & 21 & 13 & \\
$\quad$ Female & 0 & 4 & $\mathbf{0 . 0 3}$ \\
Stage & & & \\
$\quad$ I & 0 & 0 & \\
II & 10 & 9 & 0.99 \\
III & 11 & 8 &
\end{tabular}

Histological grade

1 (well)

2 (moderate)

3 (poor)
2

9

10
0

17

0
0.0008

*Data (years) are presented as the mean \pm SD. All other values represent the number of patients. $\mathrm{P}$ values $<0.05$ are considered to be significant, indicated bold.

Table 3. Expression Profiles of MITF and GLI1 in Hepatocellular Carcinoma.

\begin{tabular}{ccc}
\hline \multicolumn{2}{c}{ MITF immunoreactivity } & P value \\
\cline { 2 - 3 } & positive $(n=6) \quad$ negative $(n=32)$ & \\
\hline
\end{tabular}

\section{GLI1 immunoreactivity}

$\begin{array}{llll}\text { positive }(\mathrm{n}=21) & 6 & 15 & \\ \text { negative }(\mathrm{n}=17) & 0 & 17 & \mathbf{0 . 0 2}\end{array}$

All values represent the number of patients. P values $<0.05$ are considered to be significant, indicated bold.

gesting that MITF expression may be correlated to GLI1 expression in a subset of hepatocellular carcinoma. These results are also consistent in part with the proposal that MITF expression is associated with poor prognosis in hepatocellular carcinoma. On the other hand, MITF immunoreactivity was undetectable in most of GLI1-positive hepatocellular carcinoma $(n=15,71 \%$; Table 3$)$. Thus, GLI1 may differentially influence MITF expression, depending on the activity of Hedgehog signaling pathway in hepatocellular carcinoma cells.

Expression profiles of MITF and GLI1 in cholangiocarcinoma specimens

We also analyzed the expression profiles of MITF and GLI1 in cholangiocarcinoma specimens $(n=7)$, although we were unable to perform the statistical analysis with such a small sample size (Tables 4 and 5). All of the seven cholangiocarcinoma specimens showed moderately differentiated phenotypes. MITF immunoreactivity was detected in two cholangiocarcinoma specimens $(29 \%, 2$ out of 7 specimens; Table 4), while GLI1 immunoreactivity was detected in six cholangiocarcinoma specimens $(86 \% ; 6$ out of 7 specimens; Table 5). Importantly, MITF immunoreactivity was detected only in the GLI1-positive cholangiocarcinoma (Table 6), which is similar to the finding in hepatocellular carcinoma.

Among the seven specimens (Table 6), one cholangiocarcinoma specimen was negative for both MITF and GLI1 immunoreactivities (Fig. 2A). The undetectable GLI1 expression suggests that Hedgehog signaling may be impaired in a subset of cholangiocarcinoma, because GLI1 is expressed in the normal bile duct (Kiesslich et al. 2014). On the other hand, there were two cholangiocarcinoma specimens that were positive for both MITF and GLI1 immunoreactivities (Table 6). One of the two double-positive cholangiocarcinoma specimens is shown in Fig. 2B. Notably, MITF immunoreactivities were detected in cytoplasm of cholangiocarcinoma cells, except for a cholangiocarcinoma cell with the nuclear expression (Fig. 2B). 
Table 4. MITF expression in Cholangiocarcinoma.

\begin{tabular}{lcc}
\hline & \multicolumn{2}{c}{ MITF immunoreactivity } \\
\cline { 2 - 3 } Age (years)* & positive $(\mathbf{n}=\mathbf{2})$ & negative $(\mathbf{n}=\mathbf{5})$ \\
Sex & 55 & $53 \pm 8$ \\
$\quad$ Male & & \\
$\quad$ Female & 1 & 2 \\
Stage & 1 & 3 \\
$\quad$ I & & \\
II & 0 & 0 \\
III & 0 & 2 \\
& 2 & 3
\end{tabular}

\section{Histological grade}

\begin{tabular}{lcc}
1 (well) & 0 & 0 \\
2 (moderate) & 2 & 5 \\
3 (poor) & 0 & 0 \\
\hline *Data (years) are presented as the average or the mean \pm SD. All other \\
values represent the number of patients.
\end{tabular}

Table 5. GLI1 expression in Cholangiocarcinoma.

\begin{tabular}{lcc}
\hline & \multicolumn{2}{c}{ GLI1 immunoreactivity } \\
\cline { 2 - 3 } & positive $(\mathbf{n}=\mathbf{6})$ & negative $(\mathbf{n}=\mathbf{1})$ \\
\hline Age (years)* & $53 \pm 8$ & 55 \\
Sex & & \\
$\quad$ Male & 3 & 1 \\
$\quad$ Female & 3 & 0 \\
Stage & & \\
I & 0 & 0 \\
II & 3 & 0 \\
III & 3 & 1
\end{tabular}

\section{Histological grade}

$\begin{array}{lll}1 \text { (well) } & 0 & 0 \\ 2 \text { (moderate) } & 6 & 1 \\ 3 \text { (poor) } & 0 & 0\end{array}$

*Data (years) are presented as the average or the mean \pm SD. All other values represent the number of patients.

Table 6. Expression Profiles of MITF and GLI1 in Cholangiocarcinoma.

\section{MITF immunoreactivity}

$$
\text { positive }(n=2) \quad \text { negative }(n=5)
$$

\section{GLI1 immunoreactivity}

$\begin{array}{lll}\text { positive }(n=6) & 2 & 4 \\ \text { negative }(n=1) & 0 & 1\end{array}$

All values represent the number of patients. 
A. MITF-negative and GLI1-negative cholangiocarcinoma.

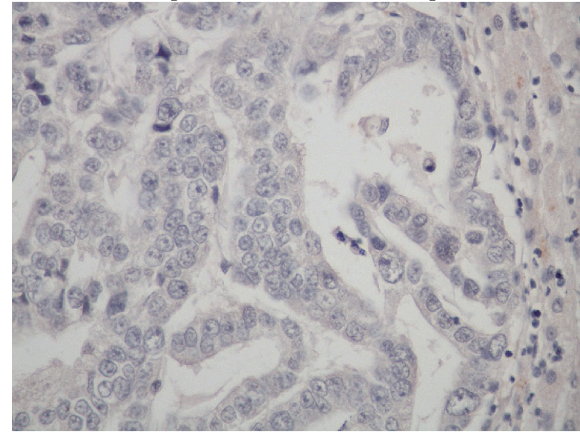

MITF

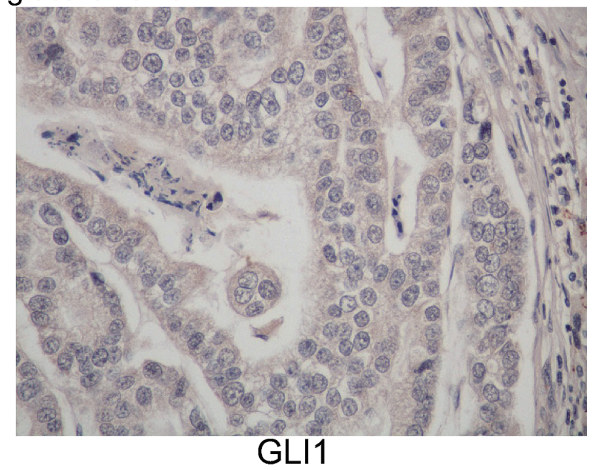

B. MITF-positive and GLI1-positive cholangiocarcinoma.
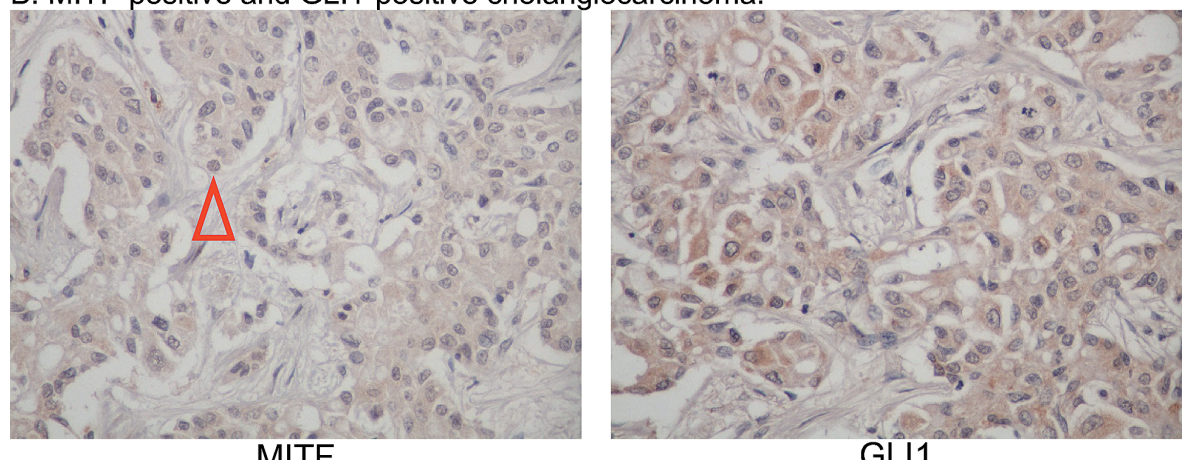

MITF

GLI1

Fig. 2. Expression profiles of immunoreactive MITF and GLI1 in cholangiocarcinoma.

(A) One cholangiocarcinoma specimen that is negative for MITF immunoreactivity (left) and GLI1 immunoreactivity (right) (magnification: $\times 400$ ). Among seven cholangiocarcinoma specimens, there was only one sample that is negative for both MITF and GLI1. (B) One cholangiocarcinoma specimen that is positive for MITF immunoreactivity (left) and GLI1 immunoreactivity (right) (magnification: $\times 400$ ). The tissue sections shown represent one of two cholangiocarcinoma specimens that are positive for both MITF and GLI1. Note that MITF immunoreactivity was detected in the nucleus of a single cholangiocarcinoma cell (indicated with red triangle). All of the cholangiocarcinoma specimens were categorized as grade 2 (moderately differentiated) (Table 4).

Differential effects of GANT61 on MITF expression in hepatic cancer cell lines

The findings with the hepatic cancer tissue array suggest that MITF may be over-expressed in subsets of hepatocellular carcinoma and cholangiocarcinoma. In particular, MITF expression is consistently associated with GLI1 expression, although MITF expression was undetectable in most of hepatocellular carcinoma and cholangiocarcinoma, irrespective of GLI1 expression. To explore the regulatory role of GLI1 for MITF expression, we analyzed the effects of GANT61 on the MITF expression in three human hepatic cancer cell lines: HepG2 hepatocellular carcinoma, HuCCT1 cholangiocarcinoma, and KKU-100 cholangiocarcinoma. GANT61 directly binds to GLI1, thereby inhibiting GLI1-mediated transcription (Agyeman et al. 2014). These cell lines were used, because we showed that the treatment for $24 \mathrm{~h}$ with cyclopamine, a Hedgehog signaling antagonist, caused the induction of MITF expression in HepG2 hepatocellular carcinoma cells and HuCCT1 cholangiocarcinoma cells, but tended to decrease the MITF level in KKU-100 cholangiocarcinoma cells, as judged by Western blot analysis (Samatiwat et al. 2016). These results suggest that MITF expression may be differentially regulated by Hedgehog signaling in hepatic cancer cells.
The treatment for $24 \mathrm{~h}$ with GANT61 at $20 \mu \mathrm{M}$ did not significantly influence the cell viability and MITF expression levels in these cell lines (data not shown). However, the treatment for $48 \mathrm{~h}$ with GANT61 at $20 \mu \mathrm{M}$ significantly decreased the viability of HepG2 cells and HuCCT1 cells, but not KKU-100 cells (Fig. 3A). Among these cell lines, HepG2 cells appeared to be more sensitive to the toxicity of GANT61, compared with HuCCT1 cells and KKU-100 cells. Importantly, the treatment for $48 \mathrm{~h}$ with GANT61 induced the MITF expression in HuCCT1 cells, but decreased MITF expression levels in HepG2 cells and KKU-100 cells (Fig. 3B). We also analyzed expression levels of HO-1 that is an enzyme responsible for physiological heme catabolism (Shibahara 2003) and also plays a cytoprotective role in cholangiocarcinoma cells (Kongpetch et al. 2012). GANT61 at 10 or $20 \mu \mathrm{M}$ induced the expression of HO-1 in HepG2 cells, HuCCT1 cells, and KKU-100 cells, which may contribute to the survival of these cancer cells. Because the effects of GANT61 were detected only after $48 \mathrm{~h}$, we suggest that GANT61 may indirectly influence the MITF expression. 
A

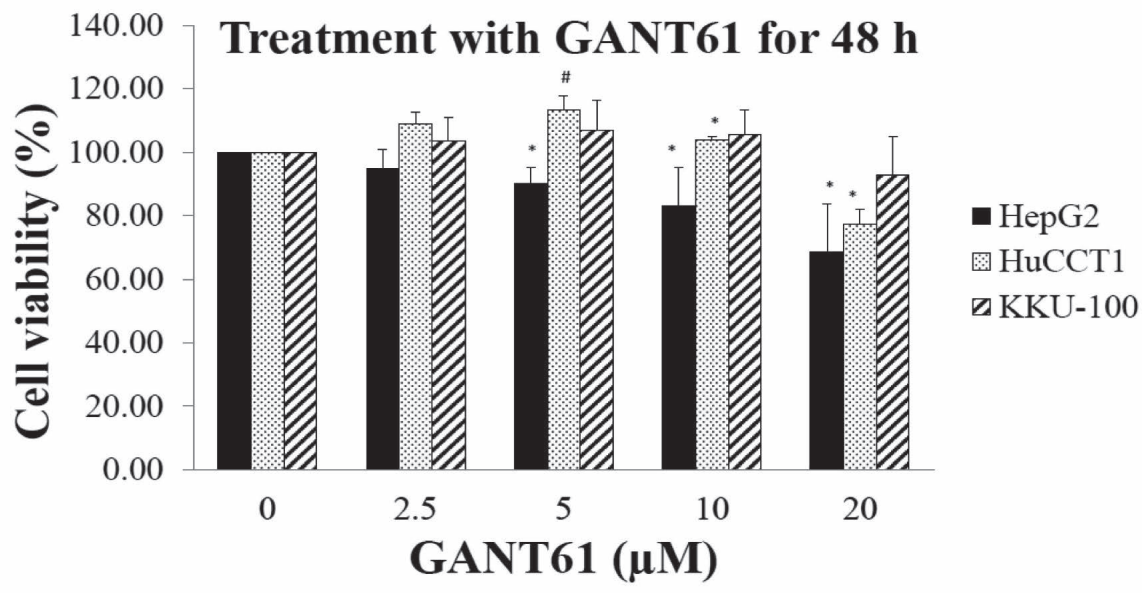

B

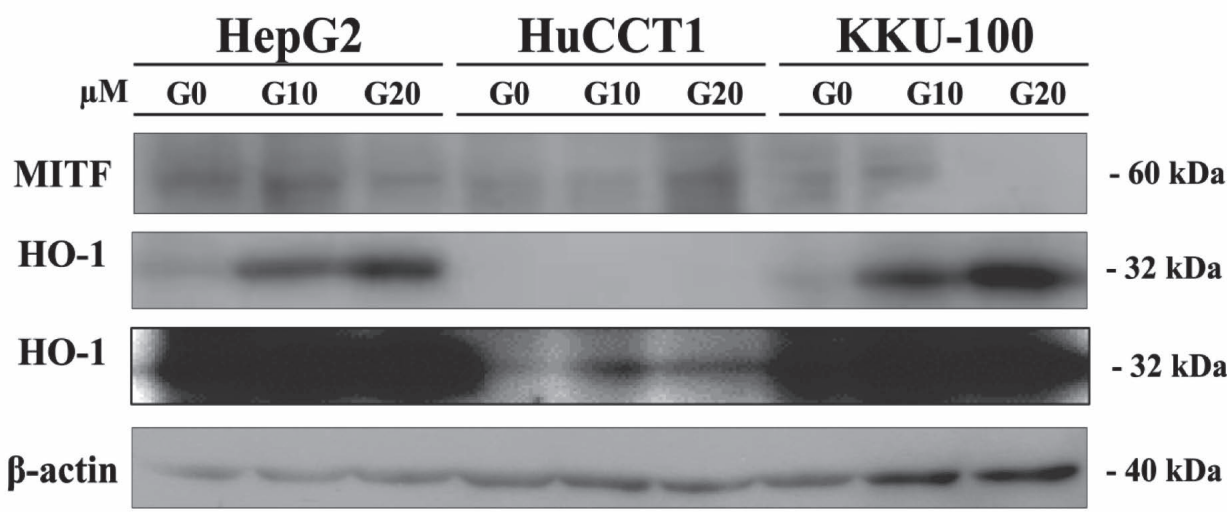

Fig. 3. Differential effects of GANT61 on MITF expression in hepatic cancer cells.

(A) The effect of GANT61 on cell viability of KKU-100, HuCCT1and HepG2 cells. Cells were incubated with GANT61 at different concentrations for $48 \mathrm{~h}$. MTT assay was used to determine the number of cell viability. Data represent mean $\pm \mathrm{SD}$ of three independent experiments. ${ }^{\#} \mathrm{P}<0.05$ compared to concurrent control. (B) Western blot analysis of MITF and HO-1. KKU-100, HuCCT1 and HepG2 cells were incubated with GANT61 (10 or $20 \mu \mathrm{M})$ or vehicle for $48 \mathrm{~h}$. The data are shown from one of two independent experiments with similar results. Each lane contained cell extracts ( $30 \mu \mathrm{g}$ protein). Under the conditions used, MITF proteins were detected as a single band of about $60 \mathrm{kDa}$. The over-exposed film $(30 \mathrm{~min})$ is also shown for visible HO-1 in HuCTT1 cells. A 10\% gel was used for detecting MITF and $\beta$-actin (top and bottom).

Induction of MITF expression by mahanine in hepatic cancer cells

The findings with the hepatic cancer tissue array and hepatic cancer cell lines suggest that MITF expression may be differentially regulated, depending on the activity of the Hedgehog signaling pathway. Among the hepatic cancer cell lines used, HuCCT1 cholangiocarcinoma cells were most resistant to cisplatin, compared with KKU-100 cholangiocarcinoma cells and HepG2 hepatocellular carcinoma cells (Samatiwat et al. 2016). Incidentally, KKU-100 cholangiocarcinoma cells were established from a Thai patient with liver fluke infection (Sripa et al. 2005). We therefore focused on mahanine, a carbazole alkaloid, that has been used as folk medicine in Thailand (Fig. 4A). Mahanine was shown to induce cell death in human cancer cells through inhibiting the autophagic degradation activity, a mechanism different from cisplatin (Utaipan et al. 2017). Treatment for $24 \mathrm{~h}$ with mahanine at $25 \mu \mathrm{M}$ significantly decreased the viability of HepG2 hepatocellular carcinoma cells and HuCCT1 and KKU-100 cholangiocarcinoma cells (Fig. 4B). Unexpectedly, MITF expression levels were increased in these dying cells treated with mahanine (Fig. 4C). Moreover, the increase in the MITF expression levels was associated with the decrease in HO-1 expression levels, the latter of which may enhance the cell toxicity of mahanine, as reported for the antitumor effect of 5-fluorouracil and gemcitabinein on cholangiocarcinoma cells (Samatiwat et al. 2015). These results suggest the possibility that mahanine is a potential reagent for the treatment of hepatic cancer.

\section{Discussion}

Cytoplasmic expression of MITF in hepatocellular carcinoma and cholangiocarcinoma

Using the human liver cancer tissue array, we show the cytoplasmic expression of MITF in subsets of hepatocellu- 

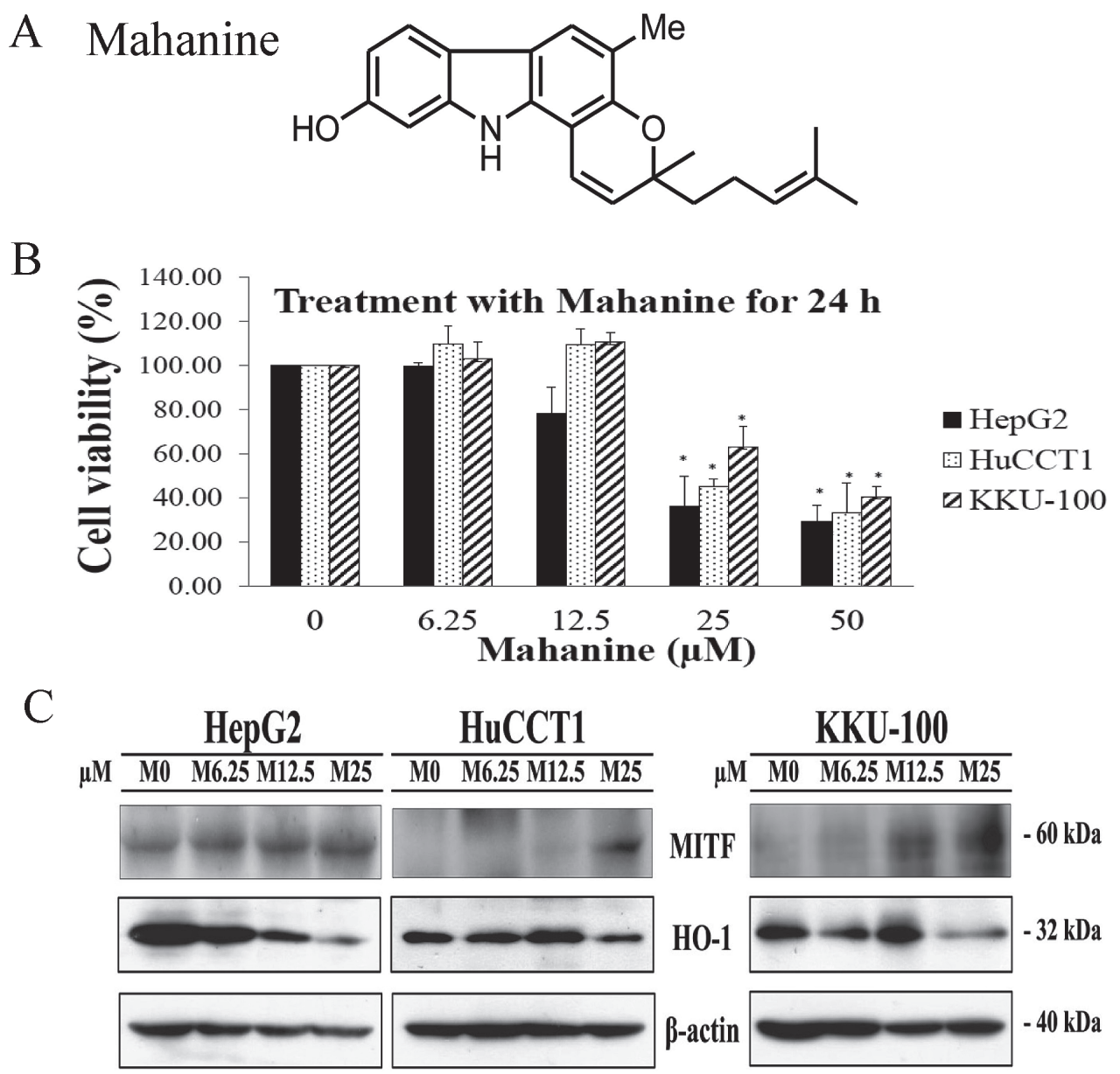

Fig. 4. Induction of MITF by mahanine in hepatic cancer cells.

(A) Structure of mahanine. (B) The effect of mahanine on viability of HepG2 hepatocellular carcinoma cells and HuCCT1 and KKU-100 cholangiocarcinoma cells. Cells were incubated with mahanine at different concentrations for $24 \mathrm{~h}$. MTT assay was used to determine the number of cell viability. Data represent mean $\pm \mathrm{SD}$ of three independent experiments. ${ }^{\#} \mathrm{P}<0.05$ compared to concurrent control. (C) Western blot analysis of MITF and HO-1. Cells were incubated with mahanine at different concentrations for $24 \mathrm{~h}$. The data are shown from one of two independent experiments with similar results. Each lane contained cell extracts (30 $\mu$ g protein). A 10\% gel was used for detecting MITF and $\beta$-actin (top and bottom).

lar carcinoma and cholangiocarcinoma specimens. Importantly, MITF is co-expressed with GLI1, a poor prognosis marker for hepatic cancer (Che et al. 2012). The clinical data also support that MITF expression is a poor prognosis marker for hepatocellular carcinoma (Table 1). However, the impact of MITF expression in the prognosis of cholangiocarcinoma remains to be investigated, due to the limited number of cholangiocarcinoma specimens. On the other hand, MITF expression is undetectable in most of hepatic cancer specimens, irrespective of GLI1 expression (Tables 3 and 6).

We are interested in the cytoplasmic expression of MITF in hepatic cancer cells, because the cytoplasmic retention of MITF was reported in pancreatic cancer cells (Perera et al. 2015). The nutritional starvation enhanced the nuclear translocation of MITF, which in turn could stimulate the processes of autophagy in pancreatic cancer (Perera et al. 2015). Thus, the cytoplasmic expression of MITF may reflect the sufficient nutrition in hepatic cancer cells, which may be beneficial for cancer cell proliferation but may be predictive of poor prognosis in cancer patients. In this context, we have shown the cytoplasmic expression of Mitf in various cell types of the mouse kidney, except for the renal tubular epithelial cells with the nuclear localization of Mitf (Ohba et al. 2016). It is therefore conceivable that cytoplasmic MITF/Mitf may exert a hitherto unknown function essential for cellular homeostasis.

Another question remains to be clarified is the identity of MITF isoform that is over-expressed in hepatocellular carcinoma and cholangiocarcinoma specimens, because MITF consists of multiple isoforms with different aminotermini (Udono et al. 2000). In this context, using the realtime RT-PCR analysis, we have shown that among MITF isoform mRNAs, only MITF-A and MITF-H mRNAs were detected in the human liver and HepG2 hepatocellular carcinoma cells (Samatiwat et al. 2016), although their expres- 
sion levels were about three-fold lower than those in HuCCT1 and KKU-100 cholangiocarcinoma cells (Samatiwat et al. 2016).

\section{Potential role of Hedgehog signaling for regulation of} MITF expression

Hedgehog signaling plays a critical role in growth of digestive tract tumors (Berman et al. 2003), and the subsequent reports showed the activation of Hedgehog signaling in hepatocellular carcinoma (Huang et al. 2006; Al-Bahrani et al. 2015; Dugum et al. 2016) and cholangiocarcinoma (Kiesslich et al. 2014; Riedlinger et al. 2014). In fact, we have shown that GLI1 immunoreactivity was detected in $55 \%$ of hepatocellular carcinoma (21/38 specimens) and $86 \%$ of cholangiocarcinoma (6/7 specimens) (see Tables 2 and 5). Furthermore, GLI1 has been shown as a poor prognosis marker for hepatic cancer (Che et al. 2012). Importantly, MITF is co-expressed with GLI1 only in subsets of hepatocellular carcinoma and cholangiocarcinoma. It is therefore conceivable that Hedgehog signaling may differentially regulate MITF expression in hepatocellular carcinoma and cholangiocarcinoma.

Here, we showed that GANT61 treatment for $48 \mathrm{~h}$ increased MITF expression levels in HuCCT1 cholangiocarcinoma cells and decreased MITF expression levels in HepG2 hepatocellular carcinoma cells and KKU-100 cholangiocarcinoma cells (see Fig. 3). Moreover, the treatment with cyclopamine caused the induction of MITF expression in HepG2 hepatocellular carcinoma cells and HuCCT1 cholangiocarcinoma cells, but tended to decrease the MITF level in KKU-100 cholangiocarcinoma cells (Samatiwat et al. 2016). These results suggest that Hedgehog signaling may down-regulate MITF expression in HuCCT1 cholangiocarcinoma cells and up-regulate MITF expression in KKU-100 cholangiocarcinoma cells. On the other hand, in HepG2 hepatocellular carcinoma cells, cyclopamine and GANT61 exerted the opposing effects on MITF expression. These results suggest that either cyclopamine or GANT61 may influence MITF expression through a mechanism that is unrelated to Hedgehog signaling.

To the best of our knowledge, there has been no report concerning the co-expression of MITF and GLI1 in any types of cancer, including hepatocellular carcinoma and cholangiocarcinoma. In this context, it was reported that MITF-M expression was inhibited in melanoma cell lines by GLI2 transcription factor that acts downstream of the Hedgehog signaling pathway (Pierrat et al. 2012). However, the expression of MITF-M mRNA was undetectable in the normal human liver, HepG2 hepatocellular carcinoma cells, and HuCCT1 and KKU-100 cholangiocarcinoma cells, in all of which MITF-A and MITF-H mRNAs are predominantly expressed (Samatiwat et al. 2016). Importantly, in addition to GLI1 (Kiesslich et al. 2014), GLI2 is also expressed in cholangiocytes of healthy subjects (Jung et al. 2007). Thus, under the physiological conditions, the Hedgehog signaling may down-regulate MITF expression in hepatocytes and/or cholangiocytes. It remains to be investigated whether GLI1 or GLI2 could influence transcription from MITF-A or MITF-H promoter (Udono et al. 2000).

Implication of the mahanine-mediated induction of MITF expression

Mahanine was proposed to induce cell death by acting as a mitochondrial complex-III inhibitor of the electron transport chain in human glioblastoma cells (Bhattacharya et al. 2014). Moreover, mahanine was reported to inhibit the autophagic degradation activity in human oral squamous cell carcinoma cells (Utaipan et al. 2017). Here we have shown that mahanine exerted the potent cell toxicity in the hepatic cancer cell lines, including HuCCT1 cells that were resistant to cisplatin (Samatiwat et al. 2016); namely, mahanine may kill hepatic cancer cells through the mechanism different from cisplatin. The decrease in HO-1 expression may also contribute to the severe cell toxicity of mahanine in hepatic cancer cells (Fig. 4). The mahaninemediated MITF induction may reflect the compensatory mechanism for restoring the cell survival. However, these unexpected findings need further experiments.

\section{Conclusion}

We have shown the cytoplasmic expression of MITF in subsets of hepatocellular carcinoma and cholangiocarcinoma specimens. Importantly, MITF immunoreactivity is detected only in the hepatic cancer specimens that also express GLI1 immunoreactivity. Subsequently, we showed that MITF expression was upregulated in dying hepatic cancer cells treated with mahanine. Thus, MITF expression level may be determined in the liver by the balance between the activity of the Hedgehog signaling pathway and the degree of the cellular stress.

\section{Acknowledgments}

Nattakarn Nooron received the Overseas Research Experience Scholarship for Graduate Student, Graduate School and Faculty of Allied Health Sciences, Chulalongkorn University, and has been supported by National Research University Project, Office of Higher Education Commission (WCU-58-041-AS), Chulalongkorn University. We are grateful to Biomedical Research Core of Tohoku University Graduate School of Medicine for allowing us to use various facilities.

\section{Conflict of Interest}

The authors declare no conflict of interest.

\section{References}

Agyeman, A., Jha, B.K., Mazumdar, T. \& Houghton, J.A. (2014) Mode and specificity of binding of the small molecule GANT61 to GLI determines inhibition of GLI-DNA binding. Oncotarget, 5, 4492-4503.

Al-Bahrani, R., Nagamori, S., Leng, R., Petryk, A. \& Sergi, C. (2015) Differential Expression of Sonic Hedgehog Protein in Human Hepatocellular Carcinoma and Intrahepatic Cholangiocarcinoma. Pathol. Oncol. Res., 21, 901-908. 
Berman, D.M., Karhadkar, S.S., Maitra, A., Montes De Oca, R., Gerstenblith, M.R., Briggs, K., Parker, A.R., Shimada, Y., Eshleman, J.R., Watkins, D.N. \& Beachy, P.A. (2003) Widespread requirement for Hedgehog ligand stimulation in growth of digestive tract tumours. Nature, 425, 846-851.

Bhattacharya, K., Bag, A.K., Tripathi, R., Samanta, S.K., Pal, B.C., Shaha, C. \& Mandal, C. (2014) Mahanine, a novel mitochondrial complex-III inhibitor induces G0/G1 arrest through redox alteration-mediated DNA damage response and regresses glioblastoma multiforme. Am. J. Cancer Res., 4, 629-647.

Che, L., Yuan, Y.H., Jia, J. \& Ren, J. (2012) Activation of sonic hedgehog signaling pathway is an independent potential prognosis predictor in human hepatocellular carcinoma patients. Chin. J. Cancer Res., 24, 323-331.

Dugum, M., Hanouneh, I., McIntyre, T., Pai, R., Aucejo, F., Eghtesad, B. \& Zein, N. (2016) Sonic hedgehog signaling in hepatocellular carcinoma: a pilot study. Mol. Clin. Oncol., 4, 369-374.

Fuse, N., Yasumoto, K., Takeda, K., Amae, S., Yoshizawa, M., Udono, T., Takahashi, K., Tamai, M., Tomita, Y., Tachibana, M. \& Shibahara, S. (1999) Molecular cloning of cDNA encoding a novel microphthalmia-associated transcription factor isoform with a distinct amino-terminus. J. Biochem., 126, 1043-1051.

Haswell-Elkins, M.R., Satarug, S. \& Elkins, D.B. (1992) Opisthorchis viverrini infection in northeast Thailand and its relationship to cholangiocarcinoma. J. Gastroenterol. Hepatol., 7, 538-548.

Hodgkinson, C.A., Moore, K.J., Nakayama, A., Steingrimsson, E., Copeland, N.G., Jenkins, N.A. \& Arnheiter, H. (1993) Mutations at the mouse microphthalmia locus are associated with defects in a gene encoding a novel basic-helix-loop-helixzipper protein. Cell, 74, 395-404.

Hozumi, H., Takeda, K., Yoshida-Amano, Y., Takemoto, Y., Kusumi, R., Fukuzaki-Dohi, U., Higashitani, A., Yamamoto, H. \& Shibahara, S. (2012) Impaired development of melanoblasts in the black-eyed white Mitf $f^{m i-b w}$ mouse, a model for auditory-pigmentary disorders. Genes Cells, 17, 494-508.

Huang, S., He, J., Zhang, X., Bian, Y., Yang, L., Xie, G., Zhang, K., Tang, W., Stelter, A.A., Wang, Q., Zhang, H. \& Xie, J. (2006) Activation of the hedgehog pathway in human hepatocellular carcinomas. Carcinogenesis, 27, 1334-1340.

Hughes, M.J., Lingrel, J.B., Krakowsky, J.M. \& Anderson, K.P. (1993) A helix-loop-helix transcription factor-like gene is located at the mi locus. J. Biol. Chem., 268, 20687-20690.

Jung, Y., McCall, S.J., Li, Y.X. \& Diehl, A.M. (2007) Bile ductules and stromal cells express hedgehog ligands and/or hedgehog target genes in primary biliary cirrhosis. Hepatology, 45, 1091-1096.

Kiesslich, T., Mayr, C., Wachter, J., Bach, D., Fuereder, J., Wagner, A., Alinger, B., Pichler, M., Di Fazio, P., Ocker, M., Berr, F. \& Neureiter, D. (2014) Activated hedgehog pathway is a potential target for pharmacological intervention in biliary tract cancer. Mol. Cell. Biochem., 396, 257-268.

Kongpetch, S., Kukongviriyapan, V., Prawan, A., Senggunprai, L., Kukongviriyapan, U. \& Buranrat, B. (2012) Crucial role of heme oxygenase- 1 on the sensitivity of cholangiocarcinoma cells to chemotherapeutic agents. PLoS One, 7, e34994.

Lauth, M., Bergstrom, A., Shimokawa, T. \& Toftgard, R. (2007) Inhibition of GLI-mediated transcription and tumor cell growth by small-molecule antagonists. Proc. Natl. Acad. Sci. USA, 104, 8455-8460.

Lee, S.T., Welch, K.D., Panter, K.E., Gardner, D.R., Garrossian, M. \& Chang, C.W. (2014) Cyclopamine: from cyclops lambs to cancer treatment. J. Agric. Food Chem., 62, 7355-7362.

Mazur, M.A., Winkler, M., Ganic, E., Colberg, J.K., Johansson, J.K., Bennet, H., Fex, M., Nuber, U.A. \& Artner, I. (2013) Microphthalmia transcription factor regulates pancreatic $\beta$-cell function. Diabetes, 62, 2834-2842.
Ohba, K., Takeda, K., Furuse, T., Suzuki, T., Wakana, S., Suzuki, T., Yamamoto, H. \& Shibahara, S. (2016) Microphthalmiaassociated transcription factor ensures the elongation of axons and dendrites in the mouse frontal cortex. Genes Cells, 21, $1365-1379$.

Ohba, K., Takeda, K., Yamamoto, H. \& Shibahara, S. (2015) Microphthalmia-associated transcription factor is expressed in projection neurons of the mouse olfactory bulb. Genes Cells, 20, 1088-1102.

Perera, R.M., Stoykova, S., Nicolay, B.N., Ross, K.N., Fitamant, J., Boukhali, M., Lengrand, J., Deshpande, V., Selig, M.K., Ferrone, C.R., Settleman, J., Stephanopoulos, G., Dyson, N.J., Zoncu, R., Ramaswamy, S., Haas, W. \& Bardeesy, N. (2015) Transcriptional control of autophagy-lysosome function drives pancreatic cancer metabolism. Nature, 524, 361-365.

Pierrat, M.J, Marsaud, V., Mauviel, A. \& Javelaud, D. (2012) Expression of microphthalmia-associated transcription factor (MITF), which is critical for melanoma progression, is inhibited by both transcription factor GLI2 and transforming growth factor- $\beta$. J. Biol. Chem., 287, 17996-18004.

Riedlinger, D., Bahra, M., Boas-Knoop, S., Lippert, S., Bradtmoller, M., Guse, K., Seehofer, D., Bova, R., Sauer, I.M., Neuhaus, P., Koch, A. \& Kamphues, C. (2014) Hedgehog pathway as a potential treatment target in human cholangiocarcinoma. J. Hepatobiliary Pancreat. Sci., 21, 607-615.

Roy, M.K., Thalang, V.N., Trakoontivakorn, G. \& Nakahara, K. (2004) Mechanism of mahanine-induced apoptosis in human leukemia cells (HL-60). Biochem. Pharmacol., 67, 41-51.

Samatiwat, P., Prawan, A., Senggunprai, L. \& Kukongviriyapan, V. (2015) Repression of Nrf2 enhances antitumor effect of 5 -fluorouracil and gemcitabine on cholangiocarcinoma cells. Naunyn Schmiedebergs Arch. Pharmacol., 388, 601-612.

Samatiwat, P., Takeda, K., Satarug, S., Ohba, K., Kukongviriyapan, V. \& Shibahara, S. (2016) Induction of MITF expression in human cholangiocarcinoma cells and hepatocellular carcinoma cells by cyclopamine, an inhibitor of the Hedgehog signaling. Biochem. Biophys. Res. Commun., 470, 144-149.

Shaib, Y. \& El-Serag, H.B. (2004) The epidemiology of cholangiocarcinoma. Semin. Liver Dis., 24, 115-125.

Shibahara, S. (2003) The heme oxygenase dilemma in cellular homeostasis: new insights for the feedback regulation of heme catabolism. Tohoku J. Exp. Med., 200, 167-186.

Shin, H.R., Oh, J.K., Masuyer, E., Curado, M.P., Bouvard, V., Fang, Y.Y., Wiangnon, S., Sripa, B. \& Hong, S.T. (2010) Epidemiology of cholangiocarcinoma: an update focusing on risk factors. Cancer Sci., 101, 579-585.

Smith, S.B., Duncan, T., Kutty, G., Kutty, R.K. \& Wiggert, B. (1994) Increase in retinyl palmitate concentration in eyes and livers and the concentration of interphotoreceptor retinoidbinding protein in eyes of vitiligo mutant mice. Biochem. J., 300 (Pt 1), 63-68.

Sripa, B., Leungwattanawanit, S., Nitta, T., Wongkham, C., Bhudhisawasdi, V., Puapairoj, A., Sripa, C. \& Miwa, M. (2005) Establishment and characterization of an opisthorchiasis-associated cholangiocarcinoma cell line (KKU-100). World J. Gastroenterol., 11, 3392-3397.

Steingrimsson, E., Moore, K.J., Lamoreux, M.L., Ferre-D'Amare, A.R., Burley, S.K., Zimring, D.C., Skow, L.C., Hodgkinson, C.A., Arnheiter, H., Copeland, N.G. \& Jenkins, N.A. (1994) Molecular basis of mouse microphthalmia ( $m i)$ mutations helps explain their developmental and phenotypic consequences. Nat. Genet., 8, 256-263.

Takeda, K., Adachi, T., Han, F., Yokoyama, S., Yamamoto, H., Hida, W. \& Shibahara, S. (2007) Augmented chemosensitivity in black-eyed white Mitf $f^{\text {mi-bw }}$ mice, lacking melanocytes. J. Biochem., 141, 327-333.

Takeda, K., Hozumi, H., Nakai, K., Yoshizawa, M., Satoh, H., Yamamoto, H. \& Shibahara, S. (2014) Insertion of long interspersed element-1 in the Mitf gene is associated with altered 
neurobehavior of the black-eyed white Mitf ${ }^{\text {ni-bw }}$ mouse. Genes Cells, 19, 126-140.

Udono, T., Yasumoto, K., Takeda, K., Amae, S., Watanabe, K., Saito, H., Fuse, N., Tachibana, M., Takahashi, K., Tamai, M. \& Shibahara, S. (2000) Structural organization of the human microphthalmia-associated transcription factor gene containing four alternative promoters. Biochim. Biophys. Acta, 1491, 205-219.

Utaipan, T., Athipornchai, A., Suksamrarn, A., Jirachotikoon, C., Yuan, X., Lertcanawanichakul, M. \& Chunglok, W. (2017) Carbazole alkaloids from Murraya koenigii trigger apoptosis and autophagic flux inhibition in human oral squamous cell carcinoma cells. J. Nat. Med., 71, 158-169.

Varjosalo, M. \& Taipale, J. (2008) Hedgehog: functions and mechanisms. Genes Dev., 22, 2454-2472.

Yasumoto, K., Takeda, K., Saito, H., Watanabe, K., Takahashi, K. \& Shibahara, S. (2002) Microphthalmia-associated transcription factor interacts with LEF-1, a mediator of Wnt signaling. EMBO J., 21, 2703-2714.

Yasumoto, K. Yokoyama, K., Shibata, K., Tomita, Y. \& Shibahara, S. (1994) Microphthalmia-associated transcription factor as a regulator for melanocyte-specific transcription of the human tyrosinase gene. Mol. Cell. Biol., 14, 8058-8070. 